\title{
Linear relations for a generalized Tutte polynomial
}

\author{
Gary Gordon \\ Department of Mathematics \\ Lafayette College \\ Easton, PA, U.S.A. \\ gordong@lafayette.edu
}

Submitted: Jul 16, 2014; Accepted: Mar 13, 2015; Published: Mar 30, 2015

Mathematics Subject Classifications: 05B35

\begin{abstract}
Brylawski proved the coefficients of the Tutte polynomial of a matroid satisfy a set of linear relations. We extend these relations to a generalization of the Tutte polynomial that includes greedoids and antimatroids. This leads to families of new identities for antimatroids, including trees, posets, chordal graphs and finite point sets in $\mathbb{R}^{n}$. It also gives a "new" linear relation for matroids that is implied by Brylawski's identities.
\end{abstract}

Keywords: Tutte polynomial, matroid, greedoid

\section{Introduction}

The Tutte polynomial was defined by Tutte [24] in the 1940's as a way to simultaneously encode the chromatic polynomial and the flow polynomial of a graph (equivalently, the chromatic polynomial of the dual when the graph is planar). The polynomial was generalized to matroids by Crapo [10] and Brylawski [4] in the 1960's and 1970's, when substantial progress was made connecting the polynomial to an impressive collection of counting problems in graphs and matroids. The expository chapter [7] remains an excellent introduction and resource for the Tutte polynomial for graphs and matroids. For an interesting account of how Tutte came to work on this polynomial, see [25].

Tutte's original definition used basis activities that depend on an ordering of the elements of the ground set of the matroid. For a matroid $M$, this formulation expresses the polynomial as a sum over the bases of the matroid: $T(M ; x, y)=\sum_{i, j \geqslant 0} b_{i, j} x^{i} y^{j}$, where $b_{i, j}$ is the number of bases of the matroid having internal activity $i$ and external activity $j$. While the definitions of internal and external activities do not concern us here, it is worth pointing out that the $b_{i, j}$ are matroid invariants, not depending on any ordering of the ground set. (An alternative approach to basis activities for the Tutte polynomial of a graph was undertaken by Bernardi [1]. His use of activities allows a different interpretation 
for the $b_{i, j}$, still dependent on an order of the edges of the graph, and does not seem to extend to matroids.)

Brylawski [5] discovered that the coefficients of the Tutte polynomial $T(M ; x, y)$ of a matroid $M$ satisfy the following collection of linear relations. (There are other boundary conditions these coefficients satisfy; Brylawski gives a basis for all such relations that we give as Theorem 34 here.) Our main theorem generalizes these relations to ranked sets, a natural generalization of matroids and greedoids based on the rank function.

Theorem 11 Let $G=(E, r)$ be a ranked set with $|E|=n$. Let $S(G ; u, v)=$ $\sum_{A \subseteq E} u^{r(E)-r(A)} v^{|A|-r(A)}$ be the corank-nullity generating function, and let

$$
T(G ; x, y)=S(G ; x-1, y-1)
$$

be the Tutte polynomial of $G$. Write $T(G ; x, y)=\sum_{i, j \in \mathbb{Z}} b_{i, j} x^{i} y^{j}$. Then

1. For all $0 \leqslant k<n$,

$$
\sum_{i=0}^{k} \sum_{j=0}^{k-i}(-1)^{j}\left(\begin{array}{c}
k-i \\
j
\end{array}\right) b_{i, j}=0 .
$$

2. For $k=n$,

$$
\sum_{i=0}^{n} \sum_{j=0}^{n-i}(-1)^{j}\left(\begin{array}{c}
n-i \\
j
\end{array}\right) b_{i, j}=(-1)^{n-r(S)} .
$$

We list the first four relations in Table 1, simplifying as much as possible. Note that the second relation listed ensures that the coefficient of $x$ equals the coefficient of $y$, provided the set has at least 2 elements. The coefficient $b_{1,0}$ is the beta invariant, and it could equally well be taken to be $b_{0,1}$. It is not difficult to prove that $b_{1,0}>0$ for a matroid if and only if $M$ is a connected matroid (assuming $M$ has at least 2 points), a result originally due to Crapo [9].

\begin{tabular}{ccc}
\hline$b_{0,0}$ & $=$ & 0 \\
$b_{0,1}$ & $=$ & $b_{1,0}$ \\
$b_{0,2}+b_{2,0}$ & $=$ & $b_{1,0}+b_{1,1}$ \\
$b_{3,0}+b_{0,2}+b_{1,2}$ & $=$ & $b_{2,0}+b_{2,1}+b_{0,3}$ \\
\hline
\end{tabular}

Table 1: Simplified versions of the first four affine relations.

A deeper connection between the Tutte polynomial and matroid connectivity was proven in [22]. The authors prove that a matroid is connected if and only if its Tutte polynomial is irreducible in the polynomial ring $\mathbb{Z}[x, y]$. The proof is based on an analysis of the identities of Theorem 34 .

Brylawski published two different proofs of these identities. In Theorem 6.6 of [5], he counts the number of flats in a matroid of a given corank and nullity. His later proof 
(Prop. 6.3.2.f of [6]) was based on more general properties involving the relation between the Tutte polynomial and the corank-nullity generating function. This latter proof is still matroid-theoretic, but it is possible to remove the dependence of the proof on the properties of the matroid rank function. This proof is the foundation for our proof of Thm. 11 here.

Our motivation in extending Brylawski's relations to non-matroidal combinatorial structures is three-fold.

1. The Tutte polynomial has important connections with combinatorial objects that do not have a matroidal structure. Extending the Tutte polynomial to greedoids and antimatroids is the focus of $[8,17,13,19]$. The polynomial and some specializations (a one-variable characteristic polynomial and the beta invariant) have revealed interesting combinatorial properties of the (non-matroidal) object under consideration. For instance, the generalized Tutte polynomial of a rooted tree is essentially a generating function for the number of rooted subtrees with $k$ edges and $l$ leaves. Then Theorem 2.8 of [17] shows that two rooted trees have the same Tutte polynomial if and only if they are isomorphic, i.e., the polynomial is a complete invariant. Thus, a rooted tree can be uniquely reconstructed from its edge-leaf subtree data. Understanding the relations satisfied by the Tutte polynomial coefficients in this context should shed light on these combinatorial structures.

2. Applying the family of identities to classes of antimatroids should be especially fruitful. In this case, we can use an expansion of the polynomial from [13] in terms of convex sets. Then we can express Tutte polynomial coefficients as a sum of $a_{i, j}$, where $a_{i, j}$ is the number of convex sets of size $i$ with exactly $j$ "interior" elements. We interpret "interior" in a variety of ways, depending on the antimatroid under consideration. This allows us to develop new identities for a variety of combinatorial objects that do not form a matroid in a natural way, including finite subsets of $\mathbb{R}^{n}$, posets (the double shelling poset antimatroid), trees, and chordal graphs (the simplicial shelling antimatroid). One example of such an identity is the following:

Corollary 33 Let $S$ be a finite subset of $\mathbb{R}^{n}$. Let $\mathcal{C}_{1}$ be the collection of all convex sets with exactly one interior point. Then

$$
\sum_{C \in \mathcal{C}_{1}}(-1)^{|C|}=(-1)^{n}|\operatorname{int}(S)|
$$

3. In a larger sense, this work attempts to isolate the properties of the Tutte polynomial that depend on the underlying matroid structure. Some of the elementary properties the coefficients satisfy for matroids are not true in our more general setting. For instance, $b_{i, j}<0$ is possible for greedoids, something that is impossible for matroids (where the $b_{i, j}$ count the number of bases with certain activities). This is made explicit in Example 39. 
Our approach also leads to a "new" result for matroids, and what appears to be a new combinatorial identity. The matroid result is the identity for the case $k=n$, given in Cor. 35:

Corollary 35 Let $M$ be a rank $r$ matroid on $n$ points. Then if $T(M)=\sum b_{i, j} x^{i} y^{j}$,

$$
\sum_{i=0}^{n} \sum_{j=0}^{n-i}(-1)^{j}\left(\begin{array}{c}
n-i \\
j
\end{array}\right) b_{i, j}=(-1)^{n-r}
$$

This property of the coefficients of the Tutte polynomial follows from the relations found by Brylawski. Indeed, Brylawski shows that the relations of Theorem 34 form a basis for all linear relations the coefficients satisfy, so the above relation must follow from those listed in Theorem 34. But this appears not to have been noticed before.

A byproduct of our approach is a combinatorial identity Cor. 14 .

Corollary 14: For all $k, n \geqslant 0$,

$$
\sum_{i=0}^{n} \sum_{j=0}^{n-i}(-1)^{i}\left(\begin{array}{c}
n-i \\
j
\end{array}\right)\left(\begin{array}{l}
k \\
i
\end{array}\right)\left(\begin{array}{l}
k \\
j
\end{array}\right)=1 .
$$

It is a straightforward exercise to prove this (using Vandermonde convolution, for example), but it may also be new. Our proof follows from one of the lemmas (Lemma 13) we use to prove the main theorem.

The paper is structured as follows. We introduce the definitions and fundamental results we will need in Section 2. The main result of the paper, Theorem 11, is proven in Section 3. The proof follows from several lemmas, but the logical dependence is straightforward. Section 4 gives interpretations of these identities for antimatroids, concentrating specifically on trees, posets, chordal graphs and finite subsets of $\mathbb{R}^{n}$. We give examples and counterexamples in Section 5. Several examples demonstrate how much the identities given here determine the Tutte polynomial of a matroid or greedoid, but also show that the Tutte polynomial does not distinguish matroids from greedoids. We conclude with some possible directions for future research in Section 6.

The author is indebted to Thomas Brylawski (1944 - 2007), who has influenced and inspired much of this work. A close read of [6] continues to reveal ideas that deserve deeper exploration.

\section{Definitions and preliminary results}

Many of the definitions and preliminary results we will need appear in [16]; see that reference for proofs.

Definition 1. A ranked set is a set $E$ with a rank function $r$. We write $G=(E, r)$, where the function $r: E \rightarrow \mathbb{Z}$ satisfies 
(R0) $r(\emptyset)=0[$ normalization $]$

(R1) $r(A) \leqslant r(E)$ for all $A \subseteq E[$ rank $E$ maximum $]$

(R2) $r(A) \leqslant|A|$ for all $A \subseteq E$ [subcardinality]

We define the generalized Tutte polynomial $T(G ; x, y)$ using the rank function:

Definition 2. Let $G=(E, r)$ be a ranked set. Then the generalized Tutte polynomial is

$$
T(G ; x, y)=\sum_{A \subseteq E}(x-1)^{r(E)-r(A)}(y-1)^{|A|-r(A) \mid} .
$$

Properties (R1) and (R2) ensure the generating function we use to define $T(G ; x, y)$ is, in fact, a polynomial. Ranked sets generalize matroids and greedoids. Matroids and greedoids can be defined as ranked sets whose rank functions satisfy properties more restrictive than (R0), (R1) and (R2).

Definition 3. A matroid $M$ is a pair $(E, r)$ where $E$ is a finite set and $r: 2^{E} \rightarrow \mathbb{Z}^{+} \cup\{0\}$ such that:

1. $r(\emptyset)=0[$ normalization $]$

2. $r(A) \leqslant r(A \cup p) \leqslant r(A)+1$ [unit rank increase]

3. $r(A \cap B)+r(A \cup B) \leqslant r(A)+r(B)$ [semimodularity]

A standard reference for matroids is Oxley's text [23]. Greedoids were introduced by Korte and Lovasz [20] as a generalization of matroids in an attempt to isolate structures where the greedy algorithm always produces an optimal solution. An extensive introduction appears in [2]. Although there are fewer axiomatizations of greedoids than there are of matroids, it is also possible define greedoids from a rank function.

Definition 4. A greedoid $G$ is a pair $(E, r)$ where $E$ is a finite set and $r: 2^{E} \rightarrow \mathbb{Z}^{+} \cup\{0\}$ such that:

1. $r(\emptyset)=0[$ normalization $]$

2. $r(A) \leqslant r(A \cup\{p\})[$ increasing]

3. $r(A) \leqslant|A|[$ subcardinality $]$

4. If $r(A)=r\left(A \cup p_{1}\right)=r\left(A \cup p_{2}\right)$, then $r\left(A \cup\left\{p_{1}, p_{2}\right\}\right)=r(A)$. [local semimodularity]

Definition 5. Let $G=(E, r)$ be a ranked set, and define duality and also deletion and contraction as follows:

- Duality $G^{*}=\left(E, r^{*}\right)$, where $r^{*}(A):=|A|+r(E-A)-r(E)$.

- Deletion $G-p=\left(E-p, r^{\prime}\right)$, where $r^{\prime}(A)=r(A)$ for all $A \subseteq E-p$. 
- Contraction $G / p:=\left(G^{*}-p\right)^{*}$.

These generalize the usual matroid definitions. Note that $r^{*}(E)=|E|-r(E)$. This definition of duality also has the involution property: $\left(G^{*}\right)^{*}=G$.

The next result is useful for computing the rank of a subset in $G / p$, and will also be needed in our proof of a deletion-contraction recursion for the Tutte polynomial (Theorem $7(1)$ ). For clarity, we may denote the rank function of $G$ by $r_{G}$.

Theorem 6 (Theorem $2.2[16]$ ). Let $G=(E, r)$, where $r: 2^{E} \rightarrow \mathbb{Z}$ satisfies $r(\emptyset)=0$. Then, for all $p \in E$ and $A \subseteq E-p$,

$$
r_{G / p}(A)=r(A \cup p)-r(p) .
$$

Applying Definition 5 to the polynomial, we get the following.

Theorem 7 (Theorem $3.1[16]$ ). Let $r: 2^{E} \rightarrow \mathbb{Z}$ be any function satisfying $r(\emptyset)=0$, and let $G=(E, r)$ be a ranked set. Then

1. Deletion-contraction: For any $p \in E$,

$$
f(G ; t, z)=t^{r(G)-r(G-p)} f(G-p ; t, z)+z^{1-r(p)} f(G / p ; t, z) .
$$

2. Duality: Let $G^{*}$ be the dual of $G$ in the sense of Definition 5. Then

$$
f\left(G^{*} ; t, z\right)=f(G ; z, t) .
$$

We will need a generalization of the matroid operation of truncation.

Definition 8. Let $G=(E, r)$ be a ranked set. Define the truncation $T(G)=\left(E, r_{T}\right)$ by specifying its rank function:

$$
\left.r_{T} A\right)= \begin{cases}r(A) & \text { if } r(A) \leqslant r(E)-1 \\ r(A)-1 & \text { if } r(A)=r(E)\end{cases}
$$

We will also need the following construction, generalizing the matroid operations of free extension and co-extension.

Definition 9. Let $G=(E, r)$ be a ranked set. Suppose $p \notin E$.

1. Free extension: $G+p$ is defined on the pair $\left(E \cup p, r^{\prime}\right)$ where

$$
r^{\prime}(A)= \begin{cases}r(A) & p \notin A, \\ r(A) & p \in A \text { and } r(A)=r(E), \\ r(A)+1 & p \in A \text { and } r(A)<r(E)\end{cases}
$$

2. Free co-extension: $G \times p=\left(G^{*}+p\right)^{*}$. 
Proposition 10. Let $G=(E, r)$ be a ranked set.

1. Rank:
(a) $r(G+p)=r(G)$
(b) $r(G \times p)=r(G)+1$,
(c) $r((G+p) / p)=r(G)-1$,
(d) $r((G \times p)-p)=r(G)+1$.

2. Deletion in free extension: $(G+p)-p=G$.

3. Contraction in free co-extension: $(G \times p) / p=G$.

Proof. 1. (a) This is immediate from the definition.

(b) Let $|E|=n$. Recall $r\left(G^{*}\right)=n-r(G)$. From Definition 5(1), we have

$$
\begin{aligned}
r(G \times p) & =r\left(\left(G^{*}+p\right)^{*}\right) \\
& =r^{*}\left(G^{*}+p\right) \\
& =n+1-r\left(G^{*}\right) \\
& =n+1-(n-r(G)) \\
& =r(G)+1 .
\end{aligned}
$$

(c) This follows from Theorem 6 .

(d) Note that $((G \times p)-p)^{*}=(G \times p)^{*} / p=\left(G^{*}+p\right) / p$. By part (c), we know $r\left(\left(G^{*}+p\right) / p\right)=r\left(G^{*}\right)-1$. Hence

$$
\begin{aligned}
r((G \times p)-p) & =n-r\left(((G \times p)-p)^{*}\right) \\
& =n-\left(r\left(G^{*}\right)-1\right) \\
& =n-(n-r(G)-1) \\
& =r(G)+1 .
\end{aligned}
$$

2. This is immediate from the definition.

3. This follows from part (2) and duality.

We remark that the proof of Prop. 10 is the same as the standard proof for matroids. 


\section{Proof of the main theorem}

In this section we prove our main theorem.

Theorem 11. Let $G=(E, r)$ be a ranked set with $|E|=n$. Let

$$
S(G ; u, v)=\sum_{A \subseteq E} u^{r(E)-r(A)} v^{|A|-r(A)}
$$

be the corank-nullity generating function, and let

$$
T(G ; x, y)=S(G ; x-1, y-1)
$$

be the Tutte polynomial of $G$. Write $T(G ; x, y)=\sum_{i, j \in \mathbb{Z}} b_{i, j} x^{i} y^{j}$. Then

1. For all $0 \leqslant k<n$,

$$
\sum_{i=0}^{k} \sum_{j=0}^{k-i}(-1)^{j}\left(\begin{array}{c}
k-i \\
j
\end{array}\right) b_{i, j}=0
$$

2. For $k=n$,

$$
\sum_{i=0}^{n} \sum_{j=0}^{n-i}(-1)^{j}\left(\begin{array}{c}
n-i \\
j
\end{array}\right) b_{i, j}=(-1)^{n-r(E)} .
$$

The proof of Theorem 11 will follow several lemmas. We begin by investigating these identities for arbitrary 2-variable polynomials under shifting operations (multiplication by $x$ or $y$ ).

Lemma 12. Let $p(x, y)=\sum_{i, j \geqslant 0} a_{i, j} x^{i} y^{j}$ be a two-variable polynomial with coefficients in a commutative ring and let $I_{k}(p(x, y))=\sum_{i=0}^{k} \sum_{j=0}^{k-i}(-1)^{j}\left(\begin{array}{c}k-i \\ j\end{array}\right) a_{i, j}$.

1. $I_{k}\left(x^{m} p(x, y)\right)=I_{k-m}(p(x, y))$ for $0 \leqslant m \leqslant k$.

2. $I_{k}(y \cdot p(x, y))=(-1) \sum_{s=0}^{k-1} I_{s}(p(x, y))$.

Proof. We prove (1) for $m=1$; the result follows by induction on $m$. Note that $x p(x, y)=$ $\sum_{i \geqslant 1, j \geqslant 0} a_{i-1, j} x^{i} y^{j}$. Then

$$
\begin{aligned}
I_{k}(x p(x, y)) & =\sum_{i=0}^{k} \sum_{j=0}^{k-i}(-1)^{j}\left(\begin{array}{c}
k-i \\
j
\end{array}\right) a_{i-1, j} \\
& \left.=\sum_{i=1}^{k} \sum_{j=0}^{k-i}(-1)^{j}\left(\begin{array}{c}
k-i \\
j
\end{array}\right) a_{i-1, j} \quad \text { [since } a_{-1, j}=0 \text { for all } j\right] \\
& \left.=\sum_{i^{\prime}=0}^{k-1} \sum_{j=0}^{k-1-i^{\prime}}(-1)^{j}\left(\begin{array}{c}
k-1-i^{\prime} \\
j
\end{array}\right) a_{i^{\prime}, j} \quad \text { [setting } i^{\prime}=i-1\right] \\
& =I_{k-1}(p(x, y)) .
\end{aligned}
$$


For part (2), we first reverse the order of the sum to rewrite

$$
I_{k}(p(x, y))=\sum_{j=0}^{k} \sum_{i=0}^{k-j}(-1)^{j}\left(\begin{array}{c}
k-i \\
j
\end{array}\right) a_{i, j}
$$

and note that $y p(x, y)=\sum_{i \geqslant 0, j \geqslant 1} a_{i, j-1} x^{i} y^{j}$. Then

$$
\begin{aligned}
I_{k}(y p(x, y)) & =\sum_{j=0}^{k} \sum_{i=0}^{k-j}(-1)^{j}\left(\begin{array}{c}
k-i \\
j
\end{array}\right) a_{i, j-1} \\
& \left.=\sum_{j=1}^{k} \sum_{i=0}^{k-j}(-1)^{j}\left(\begin{array}{c}
k-i \\
j
\end{array}\right) a_{i, j-1} \quad \text { [since } a_{i,-1}=0 \text { for all } i\right] \\
& \left.=\sum_{j^{\prime}=0}^{k-1} \sum_{i=0}^{k-j^{\prime}-1}(-1)^{j^{\prime}+1}\left(\begin{array}{c}
k-i \\
j^{\prime}+1
\end{array}\right) a_{i, j^{\prime}} \quad \text { [setting } j^{\prime}=j-1\right] \\
& =(-1) \sum_{j^{\prime}=0}^{k-1} \sum_{i=0}^{k-j^{\prime}-1}(-1)^{j^{\prime}}\left(\begin{array}{c}
k-i \\
j^{\prime}+1
\end{array}\right) a_{i, j^{\prime}}
\end{aligned}
$$

Now use the identity $\left(\begin{array}{c}k-i \\ j^{\prime}+1\end{array}\right)=\sum_{s=i}^{k-1}\left(\begin{array}{c}s-i \\ j^{\prime}\end{array}\right)$ to get

$$
I_{k}(y p)=(-1) \sum_{j^{\prime}=0}^{k-1} \sum_{i=0}^{k-j^{\prime}-1}(-1)^{j^{\prime}} a_{i, j^{\prime}} \sum_{s=i}^{k-1}\left(\begin{array}{c}
s-i \\
j^{\prime}
\end{array}\right) .
$$

For a fixed $s$ between $i$ and $k-1$ in this sum, we get

$$
(-1) \sum_{j^{\prime}=0}^{k-1} \sum_{i=0}^{k-j^{\prime}-1}(-1)^{j^{\prime}}\left(\begin{array}{c}
s-i \\
j^{\prime}
\end{array}\right) a_{i, j^{\prime}}=(-1) \sum_{j^{\prime}=0}^{s} \sum_{i=0}^{s-j^{\prime}}(-1)^{j^{\prime}}\left(\begin{array}{c}
s-i \\
j^{\prime}
\end{array}\right) a_{i, j^{\prime}}=(-1) I_{s}(p(x, y))
$$

since terms where $j^{\prime}>s$ and $i>s-j^{\prime}$ give $\left(\begin{array}{c}s-i \\ j^{\prime}\end{array}\right)=0$. The result now follows by summing over $s$.

The proof of the next lemma uses Lemma 12.

Lemma 13. For $n, k \geqslant 0$, we have $I_{n}\left(((x-1)(y-1))^{k}\right)=1$.

Proof. We use induction on $n+k$. Let $C_{k}=((x-1)(y-1))^{k}$. The base cases of $n+k \leqslant 1$ are easy to check. Then

$$
\begin{aligned}
I_{n}\left(C_{k}\right) & =I_{n}\left((x y-x-y+1) C_{k-1}\right) \\
& =I_{n}\left(x y C_{k-1}\right)-I_{n}\left(x C_{k-1}\right)-I_{n}\left(y C_{k-1}\right)+I_{n}\left(C_{k-1}\right) \\
& =I_{n-1}\left(y C_{k-1}\right)-I_{n-1}\left(C_{k-1}\right)-I_{n}\left(y C_{k-1}\right)+I_{n}\left(C_{k-1}\right)
\end{aligned}
$$


by Lemma 12(1).

Now $I_{n}\left(C_{k-1}\right)=I_{n-1}\left(C_{k-1}\right)=1$ by induction. For the remaining terms, we use Lemma 12(2).

$$
I_{n-1}\left(y C_{k-1}\right)=(-1) \sum_{s=0}^{n-2} I_{s}\left(C_{k-1}\right) \text { and } I_{n}\left(y C_{k-1}\right)=(-1) \sum_{s=0}^{n-1} I_{s}\left(C_{k-1}\right) .
$$

Thus, by induction, we have $I_{n-1}\left(y C_{k-1}\right)=1-n$ and $I_{n}\left(y C_{k-1}\right)=-n$. Hence $I_{n}\left(C_{k}\right)=$ $(1-n)-1-(-n)+1=1$, as desired.

Note that $((x-1)(y-1))^{k}=\sum_{i=0}^{k} \sum_{j=0}^{k}(-1)^{i+j}\left(\begin{array}{l}k \\ i\end{array}\right)\left(\begin{array}{l}k \\ j\end{array}\right) x^{i} y^{j}$. Then Lemma 13 has the following purely combinatorial identity as a corollary.

Corollary 14. For all $k, n \geqslant 0$,

$$
\sum_{i=0}^{n} \sum_{j=0}^{n-i}(-1)^{i}\left(\begin{array}{c}
n-i \\
j
\end{array}\right)\left(\begin{array}{l}
k \\
i
\end{array}\right)\left(\begin{array}{l}
k \\
j
\end{array}\right)=1
$$

It would be of interest to find a combinatorial proof of this identity.

$I_{k}(p(x, y))$ can be computed as the trace of a matrix product. Let $M_{k}$ be the $(r+1) \times$ $(n+1)$ matrix with $(i, j)$ entry $(-1)^{j+1}\left(\begin{array}{c}k-i+1 \\ j-1\end{array}\right)$ and let $B$ be the $(n+1) \times(r+1)$ matrix of coefficients of the polynomial $p(x, y)=\sum_{i, j \geqslant 0} b_{i, j} x^{i} y^{j}$, so the $(i, j)$ entry of the matrix $B$ is $b_{i-1, j-1}$.

$$
M_{k}=\left(\begin{array}{cccc}
\left(\begin{array}{c}
k \\
0
\end{array}\right) & -\left(\begin{array}{c}
k \\
1
\end{array}\right) & \left(\begin{array}{c}
k \\
2
\end{array}\right) & \ldots \\
\left(\begin{array}{c}
k-1 \\
0
\end{array}\right) & -\left(\begin{array}{c}
k-1 \\
1
\end{array}\right) & \left(\begin{array}{c}
k-1 \\
2
\end{array}\right) & \ldots \\
\left(\begin{array}{c}
k-2 \\
0
\end{array}\right) & -\left(\begin{array}{c}
k-2 \\
1
\end{array}\right) & \left(\begin{array}{c}
k-2 \\
2
\end{array}\right) & \ldots \\
\vdots & \vdots & \vdots & \vdots
\end{array}\right) \quad B=\left(\begin{array}{cccc}
b_{0,0} & b_{1,0} & b_{2,0} & \ldots \\
b_{0,1} & b_{1,1} & b_{2,1} & \ldots \\
b_{0,2} & b_{1,2} & b_{2,2} & \ldots \\
\vdots & \vdots & \vdots & \vdots
\end{array}\right)
$$

We omit the computational proof of the next proposition.

Proposition 15. Let $M_{k}$ be the $(r+1) \times(n+1)$ matrix with $(i, j)$ entry $(-1)^{i+j}\left(\begin{array}{c}k-i+1 \\ j-1\end{array}\right)$ and let $B$ be the $(n+1) \times(r+1)$ matrix of coefficients of the Tutte polynomial with $(i, j)$ entry $b_{i-1, j-1}$. Then $I_{k}(T(G ; x, y))=\operatorname{tr}\left(M_{k} B\right)$.

We write $r$ for $r(E)$ throughout the remainder of this section, and refer to $G$ as a rank $r$ ranked set. Lemmas 16, 17 and 18 all refer to the Tutte polynomial $T(G ; x, y)=\sum b_{i, j} x^{i} y^{j}$ of a ranked set $G=(E, r)$. We first prove Theorem 11 in the $k=r$ case.

Lemma 16. ( $r=k<n$ case) Suppose $r=k$ and $r<n$. Then

$$
\sum_{i=0}^{r} \sum_{j=0}^{r-i}(-1)^{j}\left(\begin{array}{c}
r-i \\
j
\end{array}\right) b_{i, j}=0
$$


Proof. (After Brylawski [6].) Let $f(G ; u, v)=\sum_{A \subseteq E} u^{r-r(A)} v^{|A|}$ be the corank-cardinality polynomial for $G$. Then note that

$$
f(G ; u, v)=v^{r} S\left(G ; \frac{u}{v}, v\right) .
$$

Using the relation $S(G ; u, v)=T(G ; u+1, v+1)$, we get

$$
f(G ; u, v)=v^{r} T\left(G ; \frac{u}{v}+1, v+1\right) .
$$

Setting $u=1$ gives

$$
(v+1)^{n}=f(G ; 1, v)=v^{r} T\left(G ; \frac{v+1}{v}, v+1\right) .
$$

Expanding the Tutte polynomial gives

$$
\begin{aligned}
(v+1)^{n} & =v^{r} T\left(G ; \frac{v+1}{v}, v+1\right) \\
& =v^{r} \sum_{i, j \geqslant 0} b_{i, j}\left(\frac{v+1}{v}\right)^{i}(v+1)^{j} \\
& =\sum_{i, j \geqslant 0} b_{i, j} v^{r-i}(v+1)^{i+j} .
\end{aligned}
$$

Setting $y=v+1$ and expanding gives

$$
y^{n}=\sum_{i, j \geqslant 0} b_{i, j}(y-1)^{r-i} y^{i+j}=\sum_{i, j \geqslant 0} b_{i, j} \sum_{k=0}^{r-i}(-1)^{k}\left(\begin{array}{c}
r-i \\
k
\end{array}\right) y^{r+j-k} .
$$

Thus,

$$
y^{n-r}=\sum_{i, j \geqslant 0} b_{i, j} \sum_{k=0}^{r-i}(-1)^{k}\left(\begin{array}{c}
r-i \\
k
\end{array}\right) y^{j-k} .
$$

This is a Laurent polynomial identity, so the constant term on the right-hand side (obtained by setting $k=j$ ) is identically zero since $r<n$. This gives

$$
\sum_{i=0}^{r} \sum_{j=0}^{r-i}(-1)^{j}\left(\begin{array}{c}
r-i \\
j
\end{array}\right) b_{i, j}=0
$$

(The upper limit for the index $i$ can be taken as $r$ since $b_{i, j}=0$ for $i>r$.)

We now use induction to prove the $k>r$ case of Theorem 11 . 
Lemma 17. $(r<k<n$ case) Suppose $r<k<n$. Then

$$
\sum_{i=0}^{k} \sum_{j=0}^{k-i}(-1)^{j}\left(\begin{array}{c}
k-i \\
j
\end{array}\right) b_{i, j}=0 .
$$

Proof. We use induction on $k-r$. If $k=r$, then the result is given by Lemma 16 . Now assume $k>r$, set $m=k-r$ and the result is true for all $G$ with $k-r<m$. Let $G^{\prime}=G \times p$ be the free co-extension of $G$. Then, from Prop. 10, we have $G^{\prime} / p=G$, so

$$
T(G ; x, y)=T\left(G^{\prime} ; x, y\right)-T\left(G^{\prime}-p ; x, y\right) .
$$

Since the $\rho\left(G^{\prime}\right)=\rho\left(G^{\prime}-p\right)=\rho(G)+1$, we have $k-r=m-1$ for $G^{\prime}-p$ and $G^{\prime}$, so the result holds for both $G^{\prime}$ and $G^{\prime}-p$ by induction. The result now follows for $G$.

We will need one more lemma before we can complete the proof of Theorem 11. In our proof of Lemma 16, we examined the constant term of equation (1):

$$
y^{n-r}=\sum_{i, j \geqslant 0} b_{i, j} \sum_{k=0}^{r-i}(-1)^{k}\left(\begin{array}{c}
r-i \\
k
\end{array}\right) y^{j-k} .
$$

We will examine non-constant terms from this equation to prove the next lemma.

Lemma 18. ( $k<r=n$ case) Suppose $r=n$. Then, for $k \leqslant n-1$, we have

$$
\sum_{i=0}^{k} \sum_{j=0}^{k-i}(-1)^{j}\left(\begin{array}{c}
k-i \\
j
\end{array}\right) b_{i, j}=0
$$

Proof. We use equation (1) from Lemma 16, setting $n=r$. It will be convenient to multiply both sides of this equation by $y^{n}$ :

$$
y^{n}=\sum_{i, j \geqslant 0} b_{i, j} \sum_{k=0}^{n-i}(-1)^{k}\left(\begin{array}{c}
n-i \\
k
\end{array}\right) y^{n+j-k} .
$$

As in the proof of Lemma 16, this is a polynomial identity. We examine the coefficients of $y^{m}$ for $0 \leqslant m \leqslant n-1$. Write

$$
\sum_{i, j \geqslant 0} b_{i, j} \sum_{k=0}^{n-i}(-1)^{k}\left(\begin{array}{c}
n-i \\
k
\end{array}\right) y^{n+j-k}=\sum_{m \geqslant 0} A_{m} y^{m} .
$$

List the coefficients of $y^{m}$ for $0 \leqslant m \leqslant n-1$ as a vector: $v_{A}:=\left\langle A_{0}, A_{1}, \ldots, A_{n-1}\right\rangle$ and let $v_{I}:=\left\langle I_{0}, I_{1}, \ldots, I_{n-1}\right\rangle$ be the vector of the $I_{k}$, where

$$
I_{k}=\sum_{i=0}^{k} \sum_{j=0}^{k-i}(-1)^{j}\left(\begin{array}{c}
k-i \\
j
\end{array}\right) b_{i, j} .
$$


Now let $N$ be the $n \times n$ lower triangular matrix with $N_{i, j}=(-1)^{n-i+1}\left(\begin{array}{c}n-j \\ i-j\end{array}\right)$. Note that $N^{-1}=N$. Then $N$ maps $v_{I}^{T}$ to $v_{A}^{T}$, i.e., $N v_{I}^{T}=v_{A}^{T}$. (This can be verified inductively, or by a direct expansion of equation (2).)

But, from equation (2), we have $A_{m}=0$ for all $0 \leqslant m \leqslant n-1$. Thus $N v_{I}=\overline{0}$, so $v_{I}=\overline{0}$, i.e., $I_{k}=0$ for all $0 \leqslant k \leqslant n-1$, as desired.

We note that if $r=n$ for a matroid $M$, then the matroid consists of $n$ isthmuses, so $T(M ; x, y)=x^{n}$. Hence Lemma 18 is trivial for matroids.

Example 19. We demonstrate the matrix equation used in the proof of Lemma 16 for $n=r=5$. Then we have the following relations:

$$
\begin{aligned}
A_{0}= & -b_{0,0} \\
A_{1}= & 5 b_{0,0}-b_{0,1}+b_{1,0} \\
A_{2}= & -10 b_{0,0}+5 b_{0,1}-b_{0,2}-4 b_{1,0}+b_{1,1}-b_{2,0} \\
A_{3}= & 10 b_{0,0}-10 b_{0,1}+5 b_{0,2}-b_{0,3}+6 b_{1,0}-4 b_{1,1}+b_{1,2}+3 b_{2,0}-b_{2,1}+b_{3,0} \\
A_{4}= & -5 b_{0,0}+10 b_{0,1}-10 b_{0,2}+5 b_{0,3}-b_{0,4}-4 b_{1,0}+6 b_{1,1}-4 b_{1,2}+b_{1,3}-3 b_{2,0} \\
& +3 b_{2,1}-b_{2,2}-2 b_{3,0}+b_{3,1}-b_{4,0}
\end{aligned}
$$

Since $N v_{I}=v_{A}$ and $N v_{A}=v_{I}$, we can express the $I_{k}$ as a linear combination of the coefficients $A_{i}$ for $i \leqslant k$, and vice versa.

$$
\left(\begin{array}{ccccc}
-1 & 0 & 0 & 0 & 0 \\
4 & 1 & 0 & 0 & 0 \\
-6 & -3 & -1 & 0 & 0 \\
4 & 3 & 2 & 1 & 0 \\
-1 & -1 & -1 & -1 & -1
\end{array}\right)\left(\begin{array}{c}
I_{0} \\
I_{1} \\
I_{2} \\
I_{3} \\
I_{4}
\end{array}\right)=\left(\begin{array}{c}
A_{0} \\
A_{1} \\
A_{2} \\
A_{3} \\
A_{4}
\end{array}\right)
$$

We now prove Theorem 11.

Proof Theorem 11. 1. Assume $k<n$. We use a double induction, first on $k$, and, for fixed $k$, on $n-k$. The result is trivial for $k=0$ and $n>0$.

Now let $k>0$ assume $I_{m}=0$ for all $m<k$ and, for fixed $m$, for all $n>m$. If $n-k=1$ (the base case for the induction on $n-k$ ), then $I_{k}=0$ by Lemma 16 (if $k=r$ ), Lemma 17 (if $k>r$ ) or Lemma 18 (if $k<r$, so $r=n$ ).

Now assume $n-k>1$ and let $e \in E$. By Theorem 7(1), we have

$$
T(G ; x, y)=(x-1)^{r(G)-r(G-e)} T(G-e ; x, y)+(y-1)^{1-r(e)} T(G / e ; x, y) .
$$

Expanding $(x-1)^{r(G)-r(G-e)}$ and $(y-1)^{1-r(e)}$ (if the exponents are positive), we can use Lemma 12 to express $I_{k}$ as a linear combination of $I_{m}$ for various values of $m \leqslant k$ where $|E-e|=n-1$. By induction on $k$ (when $m<k$ ) and $n-k$ (when $m=k$ ), the corresponding identities hold for each term on the right hand side of the recursion of equation (3), so they hold for $T(G ; x, y)$. 
2. When $n=k$, we use induction on $n-r$. Let $G^{\prime}=\left(E, r^{\prime}\right)$ be formed from $G$ by changing the rank of $E$, leaving the ranks of all other subsets alone, i.e., $r^{\prime}(A)=r(A)$ for $A \neq E$, and $r^{\prime}(E)=n$. Then it is a routine exercise to show

$$
S\left(G^{\prime} ; u, v\right)=u^{n-r} S(G ; u, v)-(u v)^{n-r}+1 .
$$

Substituting $u=x-1$ and $v=y-1$ gives

$$
T\left(G^{\prime} ; x, y\right)=(x-1)^{n-r} T(G ; x, y)-((x-1)(y-1))^{n-r}+1 .
$$

For the left-hand side of this equation, we have $r\left(G^{\prime}\right)=n$, so $I_{n}\left(T\left(G^{\prime}\right)\right)=1$ (since this is the coefficient on the left-hand side of equation (1) used in Lemma 16). For the right-hand side, note that, by Lemma 12(1), we have

$$
I_{n}\left((x-1)^{n-r} T(G)\right)=\sum_{i=0}^{n-r}(-1)^{n-r-i}\left(\begin{array}{c}
n-r \\
i
\end{array}\right) I_{n-i}(T(G)) .
$$

When $i>0$, we have $I_{n-i}(T(G))=0$ by part 1 of this theorem, so

$$
I_{n}\left((x-1)^{n-r} T(G)\right)=(-1)^{n-r} I_{n}(T(G)) .
$$

Thus, $I_{n}(T(G))=(-1)^{n-r} I_{n}\left((x-1)(y-1)^{n-r}\right)=(-1)^{n-r}$ by Lemma 13 .

\section{Antimatroids}

Antimatroids are a class of greedoids of full rank whose feasible sets are closed under taking unions. See [2] for a treatment of antimatroids as a class of greedoids, or [11], where antimatroids are used as a model for generalized convexity.

Definition 20. Let $G=(E, r)$ be a greedoid with rank function $r$ and feasible sets $\mathcal{F}$. Then $G$ is an antimatroid if $r(E)=|E|$ and $F_{1} \cup F_{2} \in \mathcal{F}$ whenever $F_{1}, F_{2} \in \mathcal{F}$.

A set is convex if its complement is feasible. Then we have an expansion of the Tutte polynomial in terms of the convex sets. For $C$ convex, let ex $(C)$ be the extreme points of $C$, i.e., $p \in \operatorname{ex}(C)$ if $p \in C$ but $p \notin \overline{C-p}$, where $\bar{A}$ is the convex closure of $A$, i.e., the smallest convex set containing $A$. We write $\operatorname{int}(C):=C-\operatorname{ex}(C)$ for the interior of $C$. The interior $\operatorname{int}(C)$ has combinatorial interpretations for all the classes of antimatroids we consider here.

Theorem 21 (Theorem 2.2 [13]). Let $G$ be an antimatroid with convex sets $\mathcal{C}$. Then

$$
T(G ; x, y)=\sum_{C \in \mathcal{C}}(x-1)^{|C|} y^{|i n t(C)|} .
$$

Let $a_{i, j}$ be the number of convex sets with $i$ points, $j$ of which are interior. Expanding $(x-1)^{|C|}$ allows us to express the Tutte coefficients $b_{i, j}$ in terms of the $a_{i, j}$. 
Lemma 22. Let $G=(E, r)$ be an antimatroid with $|E|=n$ and Tutte polynomial $T(G ; x, y)=\sum b_{i, j} x^{i} y^{j}$. Then

$$
b_{i, j}=\sum_{s=i}^{n}(-1)^{s-i}\left(\begin{array}{l}
s \\
i
\end{array}\right) a_{s, j} .
$$

Combining Lemma 22 with Theorem 11 gives us the following identities that all antimatroids satisfy.

Corollary 23. Let $G=(E, r)$ be an antimatroid with $a_{i, j}$ convex sets of size $i$ and interior of size $j$.

1. For $k<n$,

$$
\sum_{i=0}^{k} \sum_{j=0}^{k-i}(-1)^{j}\left(\begin{array}{c}
k-i \\
j
\end{array}\right) \sum_{s=i}^{n}(-1)^{s-i}\left(\begin{array}{l}
s \\
i
\end{array}\right) a_{s, j}=0 .
$$

2. For $k=n$,

$$
\sum_{i=0}^{n} \sum_{j=0}^{n-i}(-1)^{j}\left(\begin{array}{c}
n-i \\
j
\end{array}\right) \sum_{s=i}^{n}(-1)^{s-i}\left(\begin{array}{l}
s \\
i
\end{array}\right) a_{s, j}=1 .
$$

The $k=0,1$ and 2 cases of Cor. 23 (1) are worth separate consideration.

- $\boldsymbol{k}=\mathbf{0}$. We say a convex set is free if it has empty interior. Let $f_{i}$ be the number of free convex sets with $i$ points, so $f_{i}=a_{i, 0}$ (and assume $f_{0}=1$ ). Then for $k=0$, the identity from Cor. 23 (1) reduces to

$$
\sum_{i=0}^{n}(-1)^{i} f_{i}=0
$$

This identity is Theorem 4.5 in [11]; Edelman and Jamison attribute this result to Lawrence. An equivalent formulation using the characteristic polynomial appears as Prop. 7 of [18].

- $\boldsymbol{k}=\mathbf{1}$. This identity gives $b_{1,0}=b_{0,1}$, i.e., the coefficient of $x$ equals the coefficient of $y$ in the Tutte polynomial. Translating to convex sets, we get:

$$
\sum_{i=0}^{n}(-1)^{i-1} i f_{i}=\sum_{i=0}^{n}(-1)^{i} a_{i, 1}
$$

The invariant on the left-hand side is $b_{1,0}$, the beta invariant, and the right-hand side is an alternating sum over convex sets with exactly one interior point. The beta invariant gives interesting combinatorial information about the antimatroid - this is the focus of [14]. We will examine this identity for several classes of antimatroids below.

We can rewrite the right hand side as a (double) sum over all interior points in $G$. 
Corollary 24. For $p \in G$, let $\mathcal{C}_{p}$ be the collection of convex sets in $G$ with unique interior point $p$. Then

$$
b_{0,1}=\sum_{i=0}^{n}(-1)^{i} a_{i, 1}=\sum_{p \in \operatorname{int}(G)} \sum_{C \in \mathcal{C}_{p}}(-1)^{|C|}
$$

This allows us to express the beta invariant as a sum over interior points of $G$.

- $\boldsymbol{k}=\mathbf{2}$ We use $b_{0,2}+b_{2,0}=b_{1,0}+b_{1,1}$. Simplifying gives

$$
\sum_{i=0}^{n}(-1)^{i}\left(\left(\begin{array}{c}
i+1 \\
2
\end{array}\right) f_{i}+i a_{i, 1}+a_{i, 2}\right)=0 .
$$

There are several equivalent formulations. This identity, and those involving higher indices, give more involved combinatorial results for the antimatroids we treat below.

Our immediate goal is to interpret the $k=1$ identity for four families of antimatroids: trees, posets, chordal graphs and finite subsets of $\mathbb{R}^{n}$. For each class, we describe the antimatroid structure by specifying the convex sets.

\subsection{Trees}

Let $T$ be a tree with edges $E$. The pruning antimatroid $G=(E, r)$ is defined on the set of edges of $T$, where the convex sets are the subtrees of $T$. An edge is interior if it is not a leaf of the subtree.

Then a convex set (subtree) is free if it has no interior edges, i.e., the edges form a star. We now give a combinatorial interpretation to the beta invariant for a tree. We omit the proof, which follows from Prop. 1.7 and Cor. 3.3 of [15] (or can be proven directly).

Proposition 25. Let $T$ be a tree with $n$ edges and $m$ interior edges. Then

$$
\sum_{i=0}^{n}(-1)^{i} i f_{i}=m \text {. }
$$

Then the $k=1$ identity translates to the following theorem.

Theorem 26. Let $T$ be a tree with $m$ interior edges and let $\mathcal{S}$ be the collection of all subtrees with exactly one interior edge. Then

$$
\sum_{S \in \mathcal{S}}(-1)^{|S|}=-m
$$

As an example, consider the tree in Fig. 1. There are nine subtrees of size 3 with exactly one interior edge, six of size 4 , and one of size 5 . Then Theorem 26 gives $\sum_{S \in \mathcal{S}}(-1)^{|S|}=$ $-9+6-1=-4$, as required.

When $k=2$, the identity involves $f_{i}\left(=a_{i, 0}\right), a_{i, 1}$ and $a_{i, 2}$. We list the values for these invariants for the tree of Fig. 1 in Table 2.

Suppressing 0-terms, the $k=2$ identity becomes

$$
\left(-f_{1}+3 f_{2}-6 f_{3}\right)+\left(-3 a_{3,1}+4 a_{4,1}-5 a_{5,1}\right)+\left(a_{4,2}-a_{5,2}+a_{6,2}\right)
$$

This reduces to $(-9+33-18)+(-27+24-5)+(6-5+1)=0$, as required. 


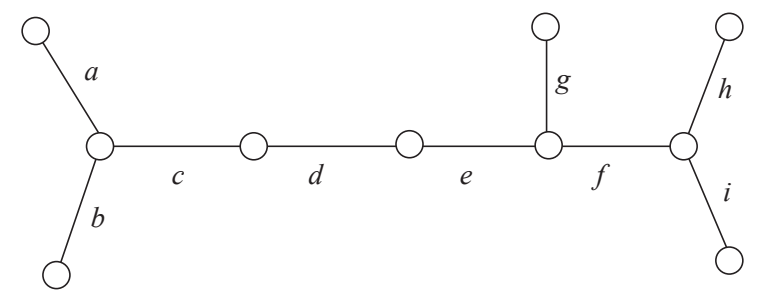

Figure 1: A tree.

\begin{tabular}{c||ccccccc} 
& $i=0$ & $i=1$ & $i=2$ & $i=3$ & $i=4$ & $i=5$ & $i=6$ \\
\hline$f_{i}=a_{i, 0}$ & 1 & 9 & 11 & 3 & 0 & 0 & 0 \\
$a_{i, 1}$ & 0 & 0 & 0 & 9 & 6 & 1 & 0 \\
$a_{i, 2}$ & 0 & 0 & 0 & 0 & 6 & 5 & 1
\end{tabular}

Table 2: Data for the tree of Fig. 1.

\subsection{Posets}

There are several ways to give a poset $P$ an antimatroid structure. We use the double shelling antimatroid. Recall a set $I$ is an order ideal in $P$ if $x \in I$ and $y \leqslant x$ implies $y \in I$. $J$ is an order filter if $x \in J$ and $y \geqslant x$ implies $y \in J$. Then a set $C$ is convex in the double shelling antimatroid associated with $P$ if there is an order ideal $I$ and an order filter $J$ such that $x \in C$ precisely when $x \geqslant y$ for all $y \in I$ and $x \leqslant z$ for all $z \in J$.

A convex set $C$ is free if it contains no chains of length greater than 2, i.e., every element of $C$ is either minimal or maximal in the poset $P-I-J$. A bottleneck in a poset is an element that is not maximal or minimal, but is comparable to every element of the poset. Then the following combinatorial characterization of the beta invariant was proven by Edelman and Reiner in [12].

Theorem 27 (Cor. 4.4 [12]). Let $P$ be a poset with $f_{i}$ free convex sets and $b$ bottlenecks. Then

$$
\sum_{i=0}^{n}(-1)^{i} i f_{i}=b .
$$

To interpret the $k=1$ identity here, we note that a convex set $C$ has exactly one interior point if $C$ is convex and there is a unique $x \in C$ with $x \notin e x(C)$. Then there is a chain of length 3 in $C$, with $x$ in the middle.

Theorem 28. Let $P$ be a poset with $b$ bottle necks and let $\mathcal{S}$ denote the set of all convex sets with exactly one interior point. Then

$$
\sum_{S \in \mathcal{S}}(-1)^{|S|}=-b
$$




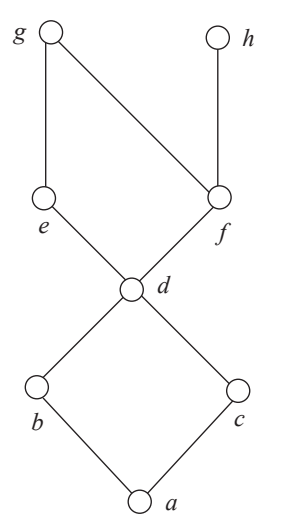

Figure 2: A poset.

As an example, consider the poset of Fig. 2. The element $d$ is a bottleneck. We list the convex sets with exactly one interior point in Table 3 . The alternating sum then gives $-4+4-1=-1$, as required.

\begin{tabular}{l|lllll} 
Size & & & & & \\
\hline 3 & $b d e$ & $b d f$ & $c d e$ & $c d f$ & $d f h$ \\
4 & $b c d e$ & $b c d f$ & $b d e f$ & $c d e f$ & defh \\
5 & bcdef & & & &
\end{tabular}

Table 3: The convex sets with exactly one interior element.

We leave full consideration of the $k=2$ case to the interested reader. Some care must be taken in determining the convex sets with exactly two interior elements, however. For instance, in the poset of Fig. 2, the chain $b<d<f<h$ is a convex set with two interior elements, but the chain $a<b<d<e$ is not, since abde is not a convex set in this antimatroid.

\subsection{Chordal graphs}

When $G$ is a chordal graph, a vertex is simplicial if its neighbors form a clique. The ground set of the simplicial shelling antimatroid is the collection of vertices of $G$. An ordered set $\left\{v_{1}, v_{2}, \ldots, v_{k}\right\}$ is shellable if $v_{1}$ is simplicial, $v_{2}$ is simplicial in $G-v_{1}, v_{3}$ is simplicial in $G-\left\{v_{1}, v_{2}\right\}$, and so on. Then we can remove the dependence on an order by defining a set to be feasible if there is some shellable ordering of the vertices. As usual, the convex sets are the complements of the feasible sets.

A set of vertices is free convex if the graph it induces is a clique in $G$. The next result gives a combinatorial interpretation to the beta invariant $b_{1,0}$. 
Theorem 29 (Theorem 5.1 [14]). Let $G$ be a chordal graph with b 2-connected blocks, and let $f_{i}$ be the number of cliques of size $i$. Then

$$
\sum_{i=0}^{n}(-1)^{i} i f_{i}=b-1 .
$$

To interpret the $k=1$ identity for chordal graphs, we need the following characterization of convex sets with a single interior vertex. A 1-sum of cliques is formed by gluing a disjoint collection of cliques together at one common vertex, as in Fig. 3. These are the convex sets we need.

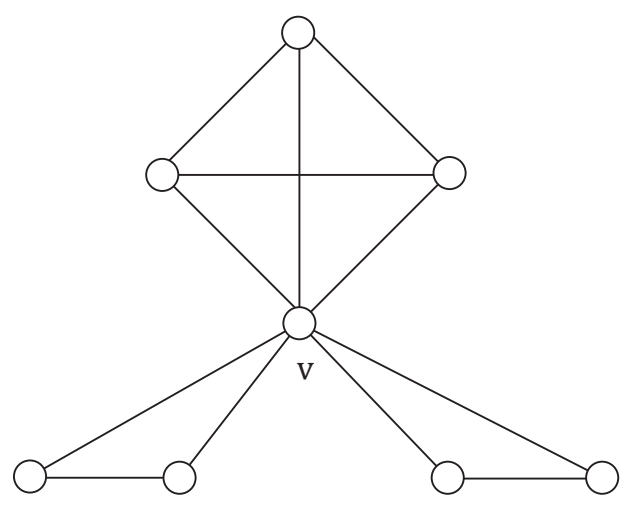

Figure 3: A 1-sum of cliques: The vertex $v$ is the unique internal vertex in the convex set in the chordal graph.

Lemma 30. Let $G$ be a chordal graph. A subset $C \subseteq V$ is convex with exactly one interior vertex if and only if the subgraph induced by $C$ is a 1-sum of cliques.

Then the $k=1$ identity takes the following form for chordal graphs.

Theorem 31. Let $G$ be a chordal graph and with $b$ blocks and let $\mathcal{S}$ denote the set of all convex sets with exactly one interior vertex. Then

$$
\sum_{S \in \mathcal{S}}(-1)^{|S|}=1-b
$$

As an example, consider the chordal graph of Fig. 4. Note that $G$ has 3 blocks. Then $d$ and $i$ are the only vertices that are unique interior vertices in any convex set. We compute $b_{0,1}$ using Cor. 24 . Then we see the vertex $d$ is in six such sets of size 3 , nine of size 4 , five of size 5 , and one of size 6 . For the vertex $i$, the corresponding counts give three sets of size 3 and two of size 4 . Then the alternating sum from Theorem 31 gives us $-9+11-5+1=-2$, as required. As with posets, some care is needed to ensure a subset of vertices gives a convex set. For instance, although the vertex set $\{d, h, i\}$ gives an induced subgraph that corresponds to a 1-sum of blocks, the set $\{d, h, i\}$ is not convex. 


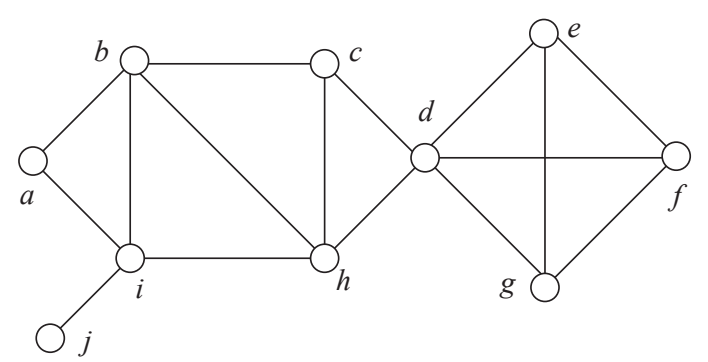

Figure 4: A chordal graph.

Trees are chordal, so Theorem 31 applies to the pruning greedoid associated to the vertices of a tree. In this context, the only free, convex sets are the single vertices and pairs of adjacent vertices. A subset of vertices has precisely one interior vertex if it corresponds to a star, where the central vertex is the interior point. (Note that this agrees with our more general interpretation given in Lemma 30.)

Thus, a vertex $v$ of degree $d$ will contribute $\sum_{i=2}^{d}(-1)^{i-1}\left(\begin{array}{c}d \\ i\end{array}\right)$ to the coefficient $b_{0,1}$. Since $\sum_{i=2}^{d}(-1)^{i-1}\left(\begin{array}{l}d \\ i\end{array}\right)=1-d$, it is easy to see that summing over all convex sets with a unique interior point gives $2-n$, which agrees with Theorem 31 since there are $n-1$ blocks (the edges of $T$ ).

\subsection{Finite point sets in Euclidean space}

Finite subsets of $\mathbb{R}^{n}$ are the prototypical examples of convex geometries, or, dually, antimatroids. A subset $C$ of a finite set $S$ is convex if $C=\bar{C} \cap S$, where $\bar{C}$ is the convex hull of $C$ in $\mathbb{R}^{n}$. In this context, interior points are straightforward. As usual, the complements of the convex sets are the feasible sets of the antimatroid.

The beta invariant counts the number of interior points, with a sign indicating the parity of the dimension of the set $S$. This theorem is the main result of [12].

Theorem 32 (Theorem $1.1[12])$. Let $S$ be a finite subset of $\mathbb{R}^{n}$. Then

$$
\sum_{i=0}^{n}(-1)^{i} i f_{i}=(-1)^{n-1}|\operatorname{int}(S)| .
$$

Combining Theorem 32 with the $k=1$ identity gives us the next result.

Corollary 33. Let $S$ be a finite subset of $\mathbb{R}^{n}$. Let $\mathcal{C}_{1}$ be the collection of all convex sets with exactly one interior point. Then

$$
\sum_{C \in \mathcal{C}_{1}}(-1)^{|C|}=(-1)^{n}|\operatorname{int}(S)|
$$

For example, the set $S$ of six points in the plane in Fig. 5 has 2 interior points, $e$ and $f$. (The point set is triangulated to help visualize the convex sets.) 


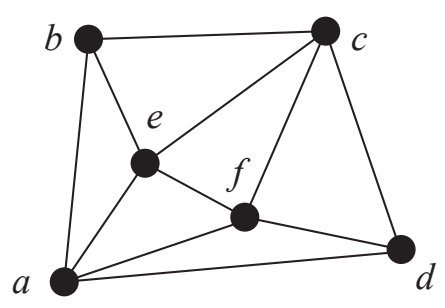

Figure 5: A finite subset of the plane.

Using the interpretation for $b_{0,1}$ given in Cor. 24, we find all convex sets that have $e$ as unique interior point, and those that have $f$. In this example, we have abce, abef and abce $f$ are the convex sets with $e$ as the unique interior point, while $f$ is the unique interior point for acdf, adef and acde $f$. This gives $b_{0,1}=4(-1)^{4}+2(-1)^{5}=2=|\operatorname{int}(S)|$.

For finite point sets, it is easier to interpret the identities for larger values of $k$. For instance, the $k=2$ identity depends on convex sets with 0,1 or 2 interior points. In our example, we have the following data (see Table 4):

\begin{tabular}{c||ccccccc} 
& $i=0$ & $i=1$ & $i=2$ & $i=3$ & $i=4$ & $i=5$ & $i=6$ \\
\hline$f_{i}=a_{i, 0}$ & 1 & 6 & 15 & 15 & 6 & 1 & 0 \\
$a_{i, 1}$ & 0 & 0 & 0 & 0 & 4 & 2 & 0 \\
$a_{i, 2}$ & 0 & 0 & 0 & 0 & 0 & 1 & 1
\end{tabular}

Table 4: Data for the point set of Fig. 5.

Then

$$
\begin{aligned}
& \sum_{i=0}^{n}(-1)^{i}\left(\left(\begin{array}{c}
i+1 \\
2
\end{array}\right) f_{i}+i a_{i, 1}+a_{i, 2}\right) \\
& =-(6)+(45)-(90)+(60+16)-(15+10+1)+(1) \\
& =0 .
\end{aligned}
$$

\section{$5 \quad$ Examples and counterexamples}

In this section, we present a series of examples to show how the identities of Theorem 11 can be used to help find the Tutte polynomial. Since matroids and greedoids (and, more generally, ranked sets) satisfy the same families of identities, it is natural to ask if the Tutte polynomial can distinguish these objects. Counterexamples are given here to show that a matroid and a greedoid (that is not a matroid) can share the same Tutte polynomial. In fact, in Example 42, we give a matroid $M^{\prime}$ and a greedoid $G$ (that is not a matroid) on the same ground set with $M^{\prime}-e=G-e$ and $M^{\prime} / e=G / e$. 
We begin by considering other relations the Tutte polynomial coefficients satisfy when the underlying object is a matroid. Here is the complete list of affine relations that form a basis for all affine relations satisfied by these coefficients.

Theorem 34 (Brylawski). Let $M$ be a matroid with no isthmuses and let $T(M ; x, y)=$ $\sum b_{i, j} x^{i} y^{j}$ be its Tutte polynomial. Let $r(M)=r$ and $|E|=n$. Then

1. $b_{i, j}=0$ for all $i>r$ and all $j>0$;

2. $b_{r, 0}=1 ; b_{r, j}=0$ for all $j>0$;

3. $b_{r-1,0}=n-r ; b_{r-1, j}=0$ for all $j>0$;

4. $b_{i, j}=0$ for all $i$ and $j$ such that $1 \leqslant i \leqslant r-2$ and $j \geqslant n-r$;

5. $b_{0, n-r}=1 ; b_{0, j}=0$ for all $j>n-r$;

6. $\sum_{i=0}^{k} \sum_{j=0}^{k-i}(-1)^{j}\left(\begin{array}{c}k-i \\ j\end{array}\right) b_{i, j}=(-1)^{n-r}$ for all $0 \leqslant k \leqslant n-1$.

Further, these identities form a basis for all affine relations satisfied by the Tutte polynomial coefficients.

Since these identities form a basis for all affine relations satisfied by the Tutte polynomial coefficients, they also generate the "new" relation of Corollary 35. We do not believe this identity has been explicitly stated before, however.

Corollary 35. Let $M$ be a rank $r$ matroid on $n$ points. Then if $T(M)=\sum b_{i, j} x^{i} y^{j}$,

$$
\sum_{i=0}^{n} \sum_{j=0}^{n-i}(-1)^{j}\left(\begin{array}{c}
n-i \\
j
\end{array}\right) b_{i, j}=(-1)^{n-r} .
$$

As an example of the $k=n$ identity, consider the two uniform matroids $U_{2,4}$ and $U_{3,4}$.

Example 36. Let $M$ be the uniform matroid $U_{2,4}$. Then $T(M ; x, y)=x^{2}+2 x+2 y+y^{2}$. Recall

$$
\begin{aligned}
I_{4}(T(M))= & b_{0,0}-4 b_{0,1}+6 b_{0,2}-4 b_{0,3}+b_{0,4}+b_{1,0}-3 b_{1,1}+3 b_{1,2}-b_{1,3} \\
& +b_{2,0}-2 b_{2,1}+b_{2,2}+b_{3,0}-b_{3,1}+b_{4,0}
\end{aligned}
$$

Then $I_{4}(T(M))=-8+6+2+1=1$, as required by the theorem since $n-r$ is even.

For $M=U_{3,4}$, we have $T(M)=x^{3}+x^{2}+x+y$. This time, we find $I_{4}(T(M))=$ $-4+1+1+1=-1$, since $n-r$ is odd.

The next set of examples examines three different rank 3, cardinality 5 ranked sets (one matroid and two greedoids). 
Example 37. Let $M$ be a rank 3 matroid on 5 points. Using all 6 of the relations of Theorem 34 forces $T(M)=x^{3}+2 x^{2}+b_{1,0} x+b_{1,1} x y+b_{0,1} y+y^{2}$, where $b_{1,0}, b_{0,1}$ and $b_{1,1}$ are undetermined. Then the simplified relations $I_{k}=0$ for $k \leqslant 4$ are:

- $I_{0}: b_{0,0}=0$.

- $I_{1}: b_{0,1}=b_{1,0}$.

- $I_{2}: b_{1,1}+b_{1,0}=3$.

- $I_{3}: 2 b_{1,1}+2 b_{1,0}=6$.

- $I_{4}: 3 b_{1,1}+3 b_{1,0}=9$.

(Note that $I_{2}, I_{3}$ and $I_{4}$ all give equivalent relations.) Now $I_{5}(T)=13-4 b_{1,0}-4 b_{1,1}$. Using $I_{2}$, this simplifies to $I_{5}(T)=1$, as required by Cor. 35. Thus, the "new" relation given is determined by the relations of Theorem 34 .

Now consider the three graphs $G, G^{\prime}$ and $G^{\prime \prime}$ of Fig. 6. Each has rank 3 on ground sets of size 5 . We give the Tutte polynomials of each, where $G$ is an unrooted graph, but $G^{\prime}$ and $G^{\prime \prime}$ are rooted graphs, and so use the branching greedoid rank function.
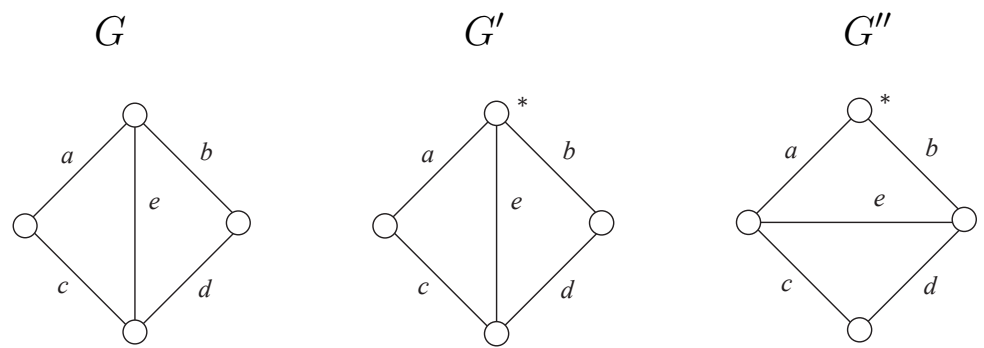

Figure 6: One unrooted and two rooted graphs for Example 37.

Then

$$
\begin{aligned}
T(G) & =x^{3}+2 x^{2}+2 x y+x+y^{2}+y \\
T\left(G^{\prime}\right) & =x^{3} y^{2}-3 x^{2} y^{2}+2 x^{2} y+x^{2}+3 x y^{2}-2 x y+3 x+3 y \\
T\left(G^{\prime \prime}\right) & =x^{3} y^{3}-3 x^{2} y^{3}+2 x^{2} y+3 x y^{3}-3 x y+4 x-y^{3}+y^{2}+4 y
\end{aligned}
$$

In Table 5 , we list the various identities $I_{0}, \ldots, I_{5}$ for each of these polynomials. Note that we use $I_{0}$ to simplify $I_{1}, I_{0}$ and $I_{1}$ to simplify $I_{2}$, and so on. We point out that the simplified matroid identity $I_{2}: b_{1,1}+b_{1,0}=3$ is false for $T\left(G^{\prime}\right)$ and $T\left(G^{\prime \prime}\right)$ (as $G^{\prime}$ and $G^{\prime \prime}$ are not matroids).

Finally, Criel Merino [21] points out that this example can be used to create a polynomial satisfying all of the matroid constraints of Theorem 34, but is not the Tutte polynomial of a matroid. In particular, let $p(x, y)=x^{3}+2 x^{2}+y^{2}+3 x y$. Then it is easy 


\begin{tabular}{|c|c|c|c|c|}
\hline$k$ & $I_{k}$ & $T(G)$ & $T\left(G^{\prime}\right)$ & $T\left(G^{\prime \prime}\right)$ \\
\hline 0 & $b_{0,0}$ & 0 & 0 & 0 \\
\hline 1 & $b_{0,1}-b_{1,0}$ & $1-1$ & $3-3$ & $4-4$ \\
\hline 2 & $b_{2,0}+b_{0,2}-b_{1,0}-b_{1,1}$ & $2+1-2-1$ & $1+0-(-2)-3$ & $0+1-(-3)+4$ \\
\hline 3 & $b_{3,0}-b_{2,1}+b_{1,2}$ & $1-0+0$ & $0-2+3$ & $0-2+0$ \\
& $-b_{0,3}-b_{2,0}+b_{0,2}$ & $-0-2+1$ & $0-1+0$ & $-(-1)-0+1$ \\
\hline
\end{tabular}

Table 5: Calculations of the identities $I_{k}$ for the three examples of Example 37.

to show that there is no matroid with this Tutte polynomial, but $p(x, y)$ satisfies all of the conditions of the theorem. It is clear that more such examples can be constructed in a similar way.

Part (6) of Theorem 34 is true in our more general setting; this is Theorem 11(1). Which of the other parts of 34 remain true? In their full generality, only part (1) is still valid.

Proposition 38. Let $G=(E, r)$ be a ranked set with Tutte polynomial $T(G ; x, y)=$ $\sum_{i, j \in \mathbb{Z}} b_{i, j} x^{i} y^{j}$. Then Theorem 34(1) remains valid: $b_{i, j}=0$ for all $i>r$ and all $j>0$.

Example 39. Let $G$ is an antimatroid with ground set $\{1,2,3\}$ and with feasible sets

$$
\{\emptyset,\{1\},\{3\},\{1,2\},\{1,3\},\{2,3\},\{1,2,3\}\} .
$$

Then $T(G ; x, y)=x^{3} y-3 x^{2} y+2 x^{2}+3 x y-x-y$. Then $r(G)=|E|=3$, and $G$ has no isthmuses. We note that $G$ is the edge pruning greedoid associated with a path on three edges. Note that

- $b_{3,0}=0$ and $b_{3,1}=1$, so Theorem $34(2)$ is no longer valid.

- $b_{2,0}=2$ and $b_{2,1}=-3$, so Theorem $34(3)$ is also false.

- $b_{1,1}=3$, so Theorem 34(4) is false, too.

When $G$ is not a matroid, we can have Tutte polynomials with negative coefficients. But this property does not distinguish the class of greedoids from matroids. The next three counterexamples examine this limitation.

Example 40. Let $G$ be a greedoid with feasible sets

$$
\mathcal{F}=\{\emptyset,\{a\},\{b\},\{c\},\{a, b\},\{a, c\},\{b, c\},\{a, d\}\} .
$$

Note that $\{d\}$ is not feasible, but $\{a, d\}$ is, so $G$ is not a matroid. This is the second truncation of the branching greedoid associated to the rooted tree in Figure 7.

Note that the feasible sets for the deletion $G-a$ are $\{\emptyset,\{b\},\{c\},\{b, c\}\}$ and the feasible sets for the contraction $G / a$ are $\{\emptyset,\{b\},\{c\},\{d\}\}$. In both cases, these are the 


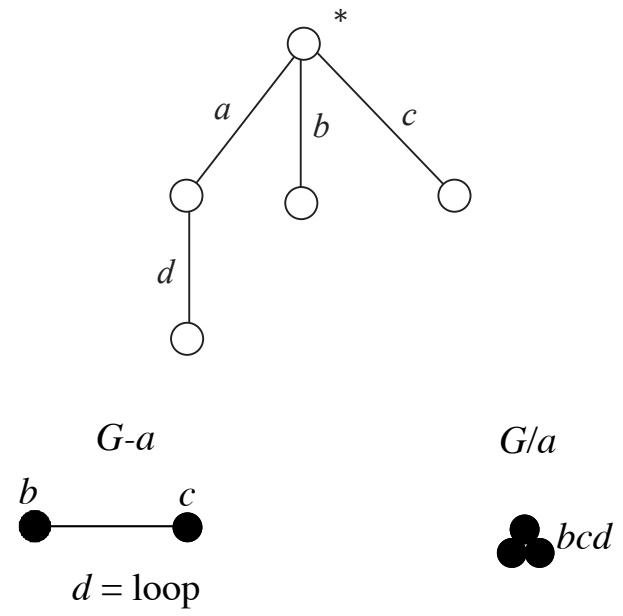

Figure 7: $G$ is the second truncation of the rooted tree above. $G-a$ and $G / a$ are both matroids.

independent sets in a matroid - see the bottom of Figure 7 for geometric depictions of these two matroids. Thus, it is possible for a greedoid $G$ that is not a matroid to have $G / x$ and $G-x$ both be matroids.

Note that $r(a)=1$ and $r(G-a)=r(G)=2$, so the recursion

$$
T(G)=(x-1)^{r(G)-r(G-a)} f(G-a)+(y-1)^{1-r(a)} f(G / a)
$$

from Theorem 7 simplifies to the familiar matroid recursion $T(G)=T(G-a)+T(G / a)$. Now $T(G-a)=x^{2} y$ since $G-a$ consists of 2 isthmuses and 1 loop, while $T(G / a)=$ $x+y+y^{2}$ since $G / a \cong U_{1,3}$. Thus $T(G ; x, y)=x^{2} y+x+y+y^{2}$.

In this case, note that there is no matroid $M$ with $M-p \cong G-a$ and $M / p \cong G-a$ for some $p \in E$. What's more, it is straightforward to check that there is no matroid with this Tutte polynomial. This gives an example of a greedoid that is not a matroid whose Tutte polynomial has all positive coefficients.

Although there is no matroid $M$ satisfying $T(M)=T(G)$ for the greedoid $G$ from Example 40, we can also construct examples where $T(M)=T(G)$ for a matroid $M$ and a greedoid $G$ that is not a matroid.

Example 41. Let $M$ be the matroid with geometric representation shown on the left in Figure 8, so $r(M)=3$ and $|E|=6$. Let $G$ be the greedoid on the ground set $E=\{a, b, c, d, e, f\}$ with feasible sets $\emptyset$, all singletons, all pairs except $a b$ and all triples except abc,ade, bef, $c d f$. One way to depict the collection of feasible triples is the set of all subsets of three labeled edges of $K_{4}$, except for the four subsets incident to a vertex, as in the graph on the right in Figure 8. Then it is routine to verify $G$ is a greedoid.

Now a computation shows

$$
T(M)=T(G)=x^{3}+x^{2} y+2 x^{2}+2 x y+3 x+y^{3}+3 y^{2}+3 y
$$




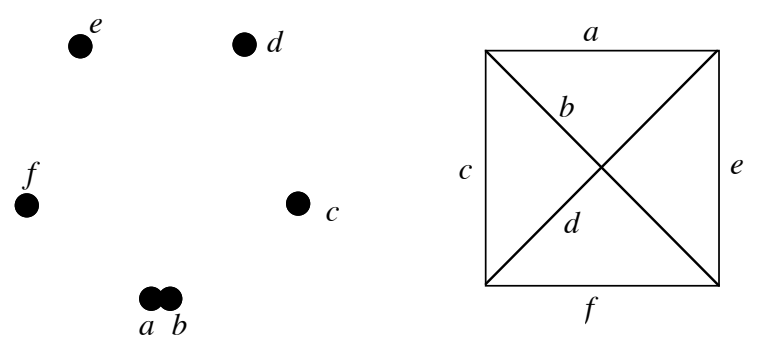

Figure 8: Left: Matroid $M$. Right: Edge labeled $K_{4}$ used to describe the feasible sets of the greedoid $G$. See Example 41.

In this case, there is no $p$ with both $G-p$ and $G / p$ matroids. Thus, if $T\left(G_{1}\right)=T\left(G_{2}\right)$ for greedoids $G_{1}$ and $G_{2}$, it need not be true that $G_{1}-a_{1} \cong G_{2}-a_{2}$ and $G_{1} / a_{1} \cong G_{2} / a_{2}$ for some $a_{1} \in E_{1}$ and $a_{2} \in E_{2}$.

In Example 40, we saw that $G-a$ and $G / a$ were both matroids, but $G-a$ contains a greedoid loop. In that example, there is no matroid $M$ with $T(G)=T(M)$. In Example 41, we found a matroid $M$ and a greedoid $G$ (where $G$ is not a matroid) with the same Tutte polynomial. Examples of two matroids (or greedoids) with the same Tutte polynomial abound, but virtually all of these arise from instances where $G_{1}-a_{1} \cong G_{2}-a_{2}$ and $G_{1} / a_{1} \cong G_{2} / a_{2}$ for some $a_{1} \in E_{1}$ and $a_{2} \in E_{2}$.

The next example combines features of Examples 40 and 41.

Example 42. Let $M^{\prime}=M-f$ be the matroid obtained by deleting $f$ from the matroid $M$ of Example 41, so $r(M)=3$ and $|E|=5$. Let $G$ be the greedoid on the ground set $E=\{a, b, c, d, e\}$ with feasible sets $\emptyset$, all singletons, all pairs except $a b$ and all triples except $a b c, a b d$ and $c d e$.

Then $M^{\prime}-e=G-e$ and $M^{\prime} / e=G / e$, so $T\left(M^{\prime}\right)=T(G)$. Hence, even when $G / p$ and $G-p$ are both matroids, with $r(G-p)=r(G)$ where $G-p$ has no loops, $G$ need not be a matroid.

We conclude with an example that examines the role of duality for greedoids. Given a ranked set $G$, we can use Def. 5(1) to define a dual object $G^{*}$ from the rank function. When $G$ is a matroid, this agrees with the usual definition of duality. But matroid duals can also be defined from the bases of $G^{*}$; these are simply the complements of the bases of $G$.

While these two approaches are identical for matroids, they are not equivalent for greedoids. Using the rank formulation for duality, Theorem 4.4 of [16] implies $G^{*}$ is also a greedoid iff $G$ is a matroid. Thus, greedoid duality from the rank function does not exist for greedoids that are not also matroids. But it is frequently the case that the basis complements of a greedoid $G$ form the bases for a different, "dual-like" greedoid (often in several distinct ways).

But even this weak form of duality does not hold for all greedoids. This is the point of our final example. 
Example 43. Exercise 8.20 of [2] asks for an example of a greedoid whose basis complements do not form the bases of any greedoid. Let $G$ be the truncation of the branching greedoid associated with the rooted graph of Fig. 9. Then $G$ has feasible sets

$$
\mathcal{F}=\{\emptyset,\{a\},\{b\},\{a, b\},\{a, c\},\{b, d\},\{a, b, c\},\{a, b, d\},\{a, c, e\},\{b, d, e\}\} .
$$

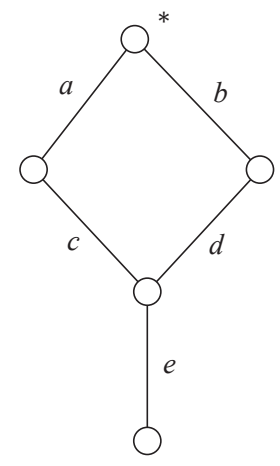

Figure 9: $G$ is the truncation of the branching greedoid associated with the rooted tree above. The collection of basis complements cannot be the bases of any greedoid.

Then the set of bases complements of $G$ is $\{\{a, c\},\{b, d\},\{c, e\},\{d, e\}\}$. To see that there is no greedoid having this collection as its bases, note that $\{a\}$ cannot be a feasible set (else feasible augmentation fails for the pair of feasible sets $\{a\}$ and $\{b, d\}$ ). Similarly, $\{c\}$ cannot be a feasible set. But then the feasible set $\{a, c\}$ is inaccessible.

We can compute the Tutte polynomial of $G$ (and recall $T\left(G^{*} ; x, y\right)=T(G ; y, x)$ from Theorem $7(2))$.

$$
T(G ; x, y)=x^{3} y^{3}-3 x^{2} y^{3}+2 x^{2} y^{2}+3 x y^{3}-4 x y^{2}+3 x y-y^{3}+3 y^{2} .
$$

Although greedoids cannot be characterized by their bases - for instance, an antimatroid has a unique basis - it is frequently the case that there is a matroid whose bases coincide with the bases of the greedoid. But this example also demonstrates that the set of bases of a greedoid do not, in general, satisfy the matroid basis properties (if they did, then, by matroid duality, their complements would, also).

\section{Future research}

There are several possibilities for further inquiry in this area. We briefly list some potential applications of Theorem 11 to other ranked sets here.

1. Other antimatroids: In Section 4, we consider four canonical classes of antimatroids: Trees, posets, chordal graphs and subsets of Euclidean space. There are other important examples of antimatroids that deserve study, however. In particular, rooted graphs and rooted digraphs (with the ground set the collection of 
all non-root vertices) form antimatroids, where vertex search is used to define the feasible sets. Then the identities of Corollary 23 should have direct combinatorial interpretations.

2. Other greedoids: Interval greedoids form a special class of greedoids that includes antimatroids. Interval greedoids are characterized by the following property:

- Suppose $A, B$ and $C$ are feasible sets with $A \subseteq B \subseteq C$. If $x \notin C$ and both $A \cup x$ and $C \cup x$ are feasible, then $B \cup x$ is also feasible.

Interpreting the relations for the Tutte polynomial for this class of greedoids might be worth pursuing, especially for the branching greedoids associated to rooted graphs and digraphs, both of which are interval greedoids, but not antimatoids.

3. Higher order relations: In Section 4, we examined the $k=0,1$, and 2 identities from Corollary 23 for four specific classes of antimatroids. It should be worthwhile to extend this analysis to other small values of $k$. In particular, interpreting the $k=3$ and $k=4$ identities for these four classes (and others) should produce new relations that hold among sets with small numbers of internal elements.

4. Further generalizations: Demi-matroids were introduced in [3], where they provide a more general setting for Wei's duality theorem for codes [26].

Definition 44. A demi-matroid is a triple $(S, r, s)$ with $S$ a finite set and rank functions $r, s: 2^{E} \rightarrow \mathbb{Z}^{+} \cup\{0\}$ satisfying

(a) $r(A) \leqslant|A|$ and $s(A) \leqslant|A|$

(b) If $A \subseteq B$, then $r(A) \leqslant r(B)$ and $s(A) \leqslant s(B)$.

(c) $|S-A|-r(S-A)=s(S)-s(A)$.

Applying Theorem 11 to these objects will produce identities that may generalize identities involving linear codes.

\section{References}

[1] O. Bernardi, "Tutte polynomial, subgraphs, orientations and sandpile model: new connections via embeddings," Electron. J. Combin. 15 (2008), 53 pp.

[2] A. Björner and G. Ziegler, "Introduction to greedoids," Matroid Applications, 284357, Encyclopedia Math. Appl., 40, (N. White ed.), Cambridge Univ. Press, Cambridge, 1992

[3] T. Britz, T. Johnsen, D. Mayhew and K Shiromoto, "Wei-type duality theorems for matroids," Des. Codes Cryptogr. 62 (2012), 331-341.

[4] T. Brylawski, "The Tutte-Grothendieck ring," Algebra Universalis 2 (1972), 375-388. 
[5] T. Brylawski, "A decomposition for combinatorial geometries," Trans. Amer. Math. Soc. 171 (1972), 235-282.

[6] T. Brylawski, "The Tutte polynomial part I: General theory," in Matroid theory and its applications, 125-275, C.I.M.E. Summer Sch., 83, Springer, Heidelberg, 2010.

[7] T. Brylawski and J. Oxley, "The Tutte polynomial and its applications," Matroid Applications, 123-225, Encyclopedia Math. Appl., 40 Cambridge Univ. Press, Cambridge, 1992.

[8] S. Chaudhary and G. Gordon, "Tutte polynomials for trees," J. Graph Theory $\mathbf{1 5}$ (1991), 317-331.

[9] H. Crapo, "A higher invariant for matroids," J. Combinatorial Theory 2 (1967), 406-417.

[10] H. Crapo, "The Tutte polynomial," Aequationes Math. 3 (1969), 211-229.

[11] P. Edelman and R. Jamison, "The theory of convex geometries," Geom. Dedicata 19 (1985), 247-270.

[12] P. Edelman and V. Reiner, "Counting the interior of a point configuration," Discrete Comput. Geom. 23 (2000), 1-13.

[13] G. Gordon, "A Tutte polynomial for partially ordered sets," J. Comb. Theory (B) 59 (1993), 132-155.

[14] G. Gordon, "A Beta invariant for greedoids and antimatroids," Electronic J. Comb. 4 (1997), R13 13 pp.

[15] G. Gordon, "Expected rank in antimatroids," Adv. in Applied Math. 32 (2004), 299318.

[16] G. Gordon, "On Brylawski's generalized duality," Math. in Comp. Sci., 6 (2012), 135-146

[17] G. Gordon and E. McMahon, "A greedoid polynomial which distinguishes rooted arborescences," Proc. Am. Math. Soc. 107 (1989), 287-298.

[18] G. Gordon and E. McMahon, "A greedoid characteristic polynomial," Contemp. Math. 197 (1996), 343-351.

[19] G. Gordon and E. McMahon, "Interval partitions and activities for the greedoid Tutte polynomial," Adv. in Applied Math. 18 (1997), 33-49.

[20] B. Korte and L. Lovász, "Mathematical structures underlying greedy algorithms," Fundamentals of computation theory (Szeged, 1981), pp. 205-209, Lecture Notes in Comput. Sci., 117 Springer, Berlin-New York, 1981.

[21] C. Merino, private communication.

[22] C. Merino, A. de Mier, M. Noy, "Irreducibility of the Tutte polynomial of a connected matroid," J. Combin. Theory Ser. B 83 (2001), 298-304.

[23] J. Oxley, Matroid Theory, Second edition, Oxford Graduate Texts in Mathematics, Oxford, 2011. 
[24] W.T. Tutte, "A ring in graph theory," Proc. Cambridge Philos. Soc. 43 (1947), 26-40.

[25] W.T. Tutte, "Graph-polynomials," Adv. in Appl. Math. 32 (2004), 5-9.

[26] V. Wei, "Generalized Hamming weights for linear codes," IEEE Trans. Inform. Theory 37 (1991), 1412-1418. 